\title{
Adaptação do modelo experimental de colestase extra-hepática em ratos jovens ${ }^{1}$
}

\author{
Ana Paula Ronquesel Battochio ${ }^{2}$, Adriane Gasparino dos Santos ${ }^{3}$, Cynthia R. Matos Silva $^{4}$, \\ Analícia S. Rocha ${ }^{5}$, Cláudio A. R. Coelho ${ }^{6}$
}

\begin{abstract}
Battochio APR, Santos AG, Silva CRM, Rocha AS, Coelho CAR. Adaptação do modelo experimental de colestase extra-hepática em ratos jovens. Acta Cir Bras [serial online] 2004 Jan-Fev;19(1). Disponível em URL: http://www.scielo.br/acb.

RESUMO - Os autores descrevem a adaptação da técnica de ligadura e ressecção do ducto biliar comum descrita na literatura, enfatizando suas vantagens na recuperação pós-cirúrgica. Este trabalho foi realizado no Laboratório Experimental do Departamento de Pediatria da FMB-UNESP.

DESCRITORES - Colestase. Cirurgia experimental. Ligadura e ressecção do ducto biliar comum.
\end{abstract}

\section{Introdução}

A colestase, caracterizada pela diminuição ou ausência do fluxo biliar, ou de algum de seus componentes, é a condição hepatobiliar mais comum no período neonatal e nos primeiros meses de vida. A deficiência da secreção de sais biliares resulta em má absorção de lipídios e vitaminas lipossolúveis ${ }^{1,2}$. O grande interesse no estudo de doenças colestáticas ocorre devido aos altos índices de mortalidade. Algumas doenças colestáticas, como a atresia de vias biliares, apresentam 100\% de mortalidade quando não tratada e é a principal indicação de transplante hepático na infância ${ }^{3}$. Com base nesses princípios, modelos experimentais em animais de laboratório que simulam colestase humana têm sido muito úteis na elucidação de diferentes etapas da patogênese da colestase $^{4}$. Várias técnicas para o desenvolvimento de colestase tem sido descritas, sobretudo em ratos, baseadas em: 1) obstrução aguda e completa do ducto biliar comum ${ }^{5}$; 2) colestase induzida por endotoxinas ${ }^{6}$; 3) colestase induzida por estinilestradiol ${ }^{6}$.
O modelo favorito para colestase extrahepática utiliza ratos submetidos à ligadura do ducto biliar comum, tendo como consequiência, na maioria das vezes, um quadro de fibrose biliar secundária ${ }^{7}$. Este modelo de colestase extra-hepática em ratos ${ }^{5}$ produz alterações estruturais e funcionais múltiplas, semelhantes às encontradas em outras causas de colestase ${ }^{8}$, pois resultam em prejuízo funcional da captação hepatocelular, excreção canalicular e transporte de sais biliares e de vários outros ânions orgânicos 9 .

Analisando e desenvolvendo o modelo adotado pela literatura ${ }^{5}$, a equipe de Hepatologia do Departamento de Pediatria UNESP, elaborou uma adaptação no manuseio deste procedimento cirúrgico.

\section{Objetivo}

Descrever a adaptação da técnica da ligadura e ressecção do ducto biliar comum (descrita por Cameron e Oakley ${ }^{5}$ ) realizada no Laboratório Experimental do Departamento de Pediatria da Faculdade de Medicina de Botucatu (FMB)-UNESP, enfatizando suas vantagens.

\section{Métodos}

O estudo da técnica da ligadura e ressecção do ducto biliar comum foi dividido basicamente em duas áreas:

\section{Teórica}

Introdução ao procedimento cirúrgico e manejo dos animais, baseado em estudos publicados $^{5}$ e na Comissão de Ensino do Colégio Brasileiro de Experimentação Animal (COBEA) ${ }^{10}$.

\section{Prática em ratos}

O procedimento cirúrgico da ligadura e ressecção do ducto biliar comum foi realizado em ratos machos (Wistar), com 21 dias de vida pós-natal (recém desmamados) e peso médio de 45 gramas. No procedimento foram utilizados:

a) Material cirúrgico:

$\varnothing$ Luvas cirúrgicas; Gazes; Fios para ligadura e sutura.

b) Instrumentos cirúrgicos:

1. Trabalho realizado no Laboratório Experimental do Departamento de Pediatria, FMB, UNESP.

2. Biomédica, doutoranda do Curso de Pós-Graduação do Departamento de Pediatria da FMB/UNESP.

3. Nutricionista, doutoranda do Curso de Pós-Graduação do Departamento de Pediatria da FMB/UNESP.

4. Nutricionista, doutoranda do Curso de Pós-Graduação do Departamento de Clínica Médica da FMB/UNESP.

5. Nutricionista, doutoranda do Curso de Pós-Graduação do Departamento de Clínica Médica da FMB/UNESP.

6. Prof. Assistente Dr., responsável pela disciplina de Hepatologia Infantil, do Departamento de Pediatria da FMB/UNESP

70 - Acta Cirúrgica Brasileira - Vol 19(1) 2004 


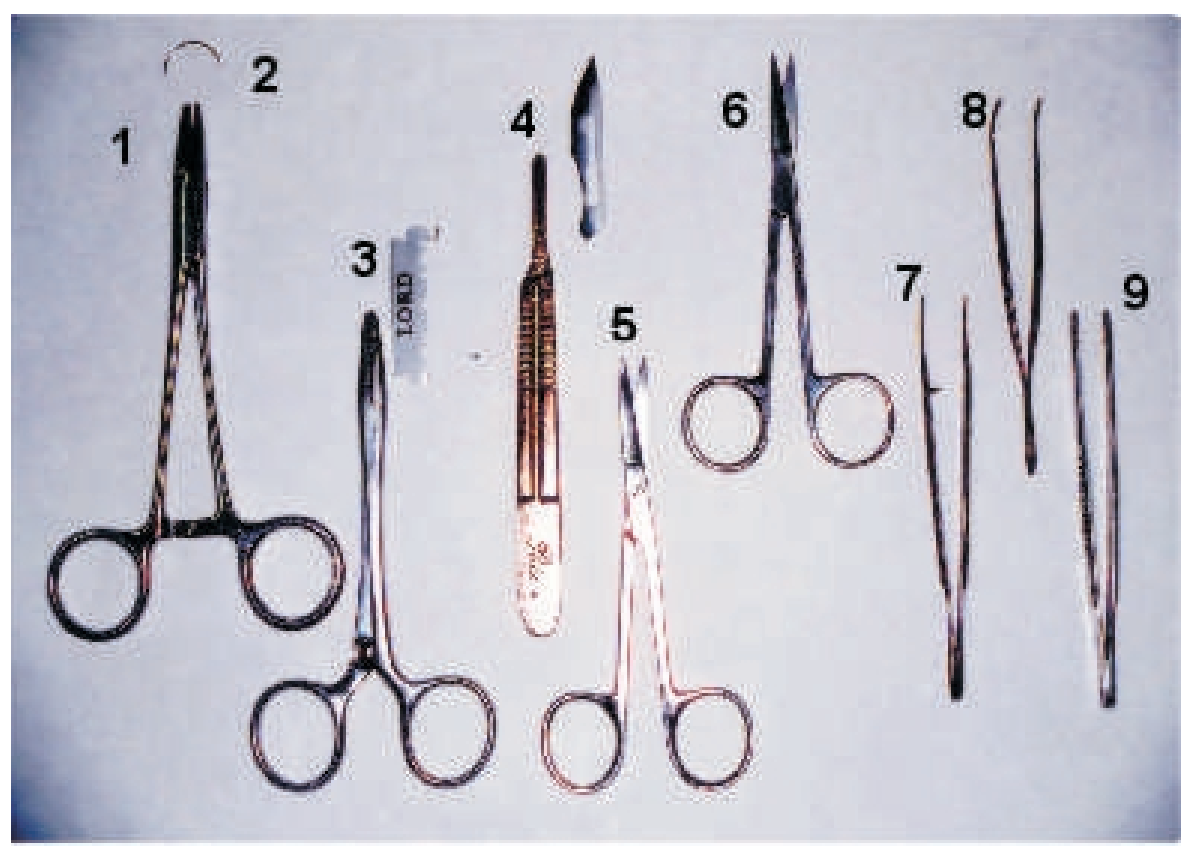

FIGURA 1 - Instrumentos cirúrgicos:1: Porta-agulhas de Mayo-Hegar; 2: Agulha curva cilíndrica; 3: Porta-agulhas de Mayo-Hegar combinado com lâmina para tricotomia; 4: Bisturi com lâmina permutável; 5: Tesoura cirúrgica reta com ponta fina; 6: Tesoura Metzenbaum; 7: Pinça reta com ponta fina; 7. Pinça curva com ponta fina; 8: Pinça anatômica.

QUADRO 1 - Manejo do animal, noções de bioterismo, técnica anestésica

Ia. Peso do animal utilizando-se a balança digital Mettler Toledo (modelo PB 3002, peso máximo $=3,100 \mathrm{~g}$, peso mínimo $=0,5 \mathrm{~g}$, sensibilidade de $0,1 \mathrm{~g}$ ).

Ib. Anestesia intraperitonial com pentobarbital na dose de $30 \mathrm{mg} / \mathrm{kg}$.

Ic. Posicionamento dos animais em decúbito dorsal horizontal na mesa operatória.

QUADRO 2 - Técnica da abordagem abdominal

IIa. Tricotomia da parede abdominal anterior.

IIb. Incisão da pele na linha mediana, desde o apêndice xifóide até a cicatriz umbilical (foto A).

IIc. Exposição das camadas musculares e a linha Alba.

IId. Incisão da linha Alba do mesmo tamanho da abertura da pele

IIe. Abertura do peritônio com tesoura e exposição dos órgãos abdominais

IIf. Exposição dos órgãos (estômago, fígado e intestino delgado) e do ducto biliar comum com abaixamento do duodeno (foto B).

QUADRO 3 - Abordagem, dissecção e ressecção do ducto biliar

IIIa. Dissecção do ducto biliar comum desde abaixo da entrada do último ducto biliar até sua porção distal, próxima ao duodeno.

IIIb. Dupla ligadura do ducto biliar comum, sendo colocadas as ligaduras o mais proximal e o mais distalmente possível (foto C).

IIIc. Ressecção da porção do ducto biliar comum entre as duas ligaduras (foto D).

IIId. Fechamento da parede abdominal com sutura contínua
Os animais foram operados pelas pósgraduandas do grupo de Hepatologia do Curso de Pediatria. O preparo pré e pósoperatório foi realizado conforme as rotinas do Biotério do Departamento de Pediatria da FMB-UNESP, sendo o projeto previamente aprovado pela Comissão de Ética na Experimentação Animal, UNESP (CEEA). A técnica da ligadura e ressecção do ducto biliar comum descrita por Cameron e Oakley $(1932)^{5}$ está apresentada nos Quadros 1, 2 e 3.

As figuras $3 \mathrm{C}$ e $3 \mathrm{D}$ diferem apenas quanto o manuseio, porém não diferem no procedimento cirúrgico (dissecção e ressecção do ducto biliar comum).

\section{Discussão}

O procedimento cirúrgico da ligadura e ressecção do ducto biliar exige alto grau de qualidade técnica, paciência, concentração, e treinamento contínuo. A qualidade técnica, importante em qualquer ato cirúrgico, se torna ainda mais importante quando se trata da realização de delicadas intervenções cirúrgicas e o sucesso é determinado pelas condições fisiológicas e metabólicas do animal.

A adaptação da técnica da ligadura e ressecção do ducto biliar comum, realizada no presente trabalho, além de demonstrar a possibilidade de se reproduzir com mais facilidade e agilidade, apresenta uma taxa de mortalidade, no momento do procedimento cirúrgico, menor do que os animais submetidos à técnica anterior ${ }^{5}$. Além disso, notou-se que o quadro pós-operatório desses animais foi muito satisfatório quanto à sua recuperação; com retorno alimentar normal mais rapidamente e diminuição do risco de infecção devido a menor manipulação do animal.

Estes dados puderam ser observados durante experimentos realizados nos últimos anos no Laboratório Experimental do Departamento de Pediatria FMB-UNESP. Estes trabalhos foram objeto de dissertações de mestrado e teses de doutorado que tiveram como objetivo determinar o efeito dos fatores moduladores das suplementações vitamínicas ${ }^{11,12}$, produtos naturais ${ }^{13}$ e dietas com baixo e alto teor de gordura ${ }^{14}$ sobre as conseqüências nutricionais e hepáticas da colestase obstrutiva crônica e também estudo da correlação e regressão múltipla entre indicadores nutricionais e hepáticos ${ }^{15}$. 


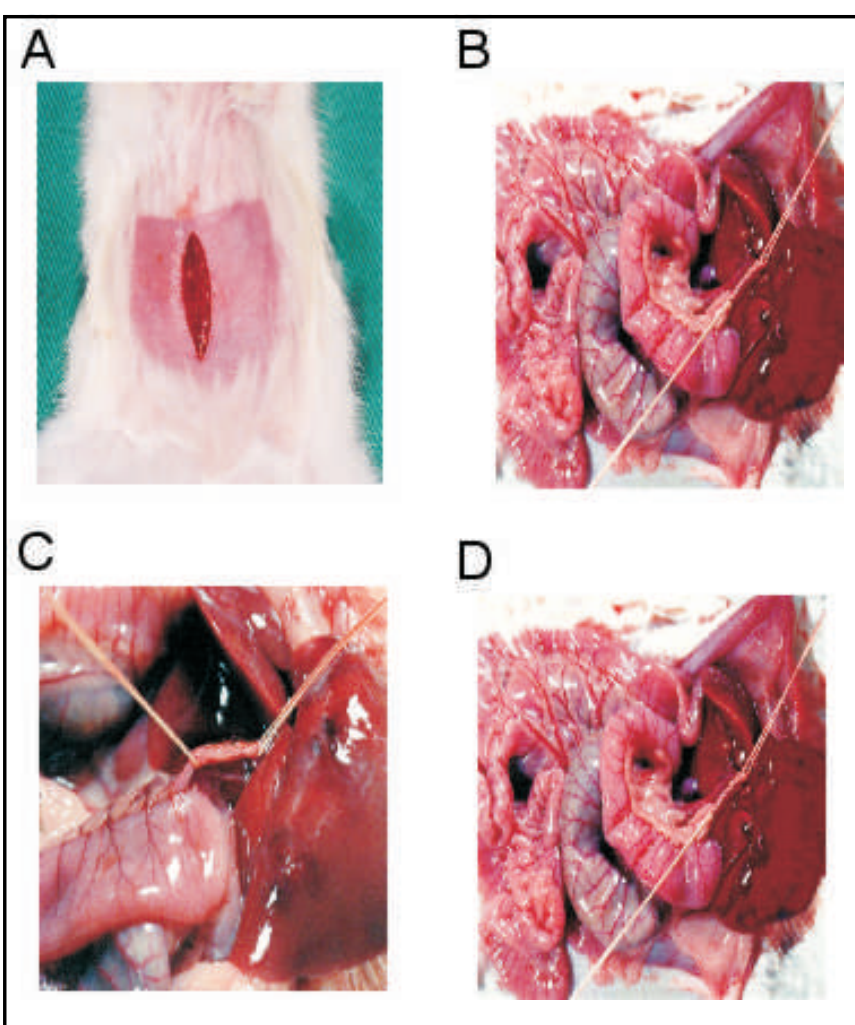

FIGURA 2 - Demonstração do procedimento cirúrgico da ligadura e ressecção do ducto biliar comum realizado segundo a técnica descrita na literatura ${ }^{5}$ realizada no Laboratório Experimental do Departamento de Pediatria, FMB-UNESP. 2A. Incisão da pele na linha mediana, desde o apêndice xifóide até a cicatriz umbilical. 2B. Exposição dos órgãos (estômago, fígado e intestino delgado) e do ducto biliar comum com abaixamento do duodeno. 2C. Dupla ligadura do ducto biliar comum, sendo colocadas às ligaduras o mais proximal e o mais distalmente possível. 2D. Ressecção da porção do ducto biliar comum entre as duas ligaduras.
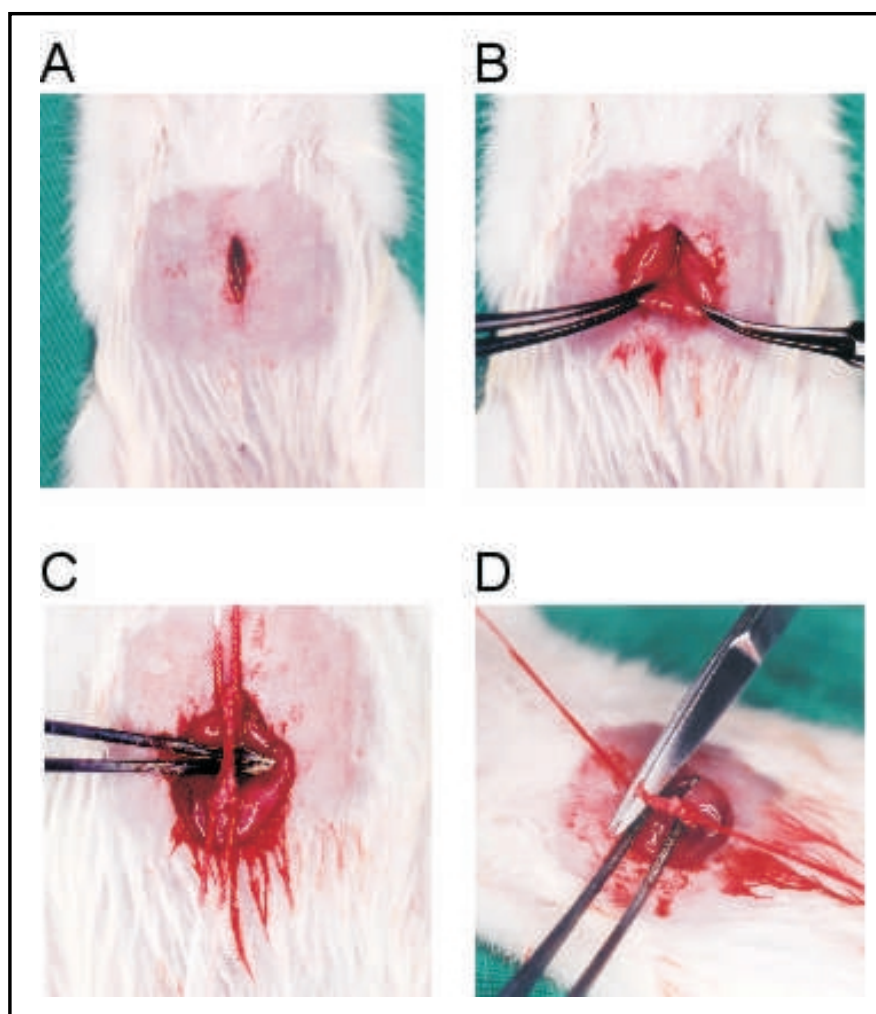

FIGURA 3 - Adaptações do procedimento cirúrgico da ligadura e ressecção do ducto biliar comum realizadas no Laboratório Experimental do Departamento de Pediatria, FMB-UNESP. A técnica adaptada (Figura 3) pelo grupo de Hepatologia do Departamento de Pediatria da UNESP consiste nos mesmos princípios descritos por Cameron e Oakley, $1932^{5}$, diferindo nos itens $2 \mathrm{~A}$ e $2 \mathrm{~B}$ da figura 2 (ou IIb e IIf do Quadro 2). 3A. Incisão da pele na linha mediana de aproximadamente $1 \mathrm{~cm}$. 3B. Abertura do peritônio com tesoura reta sem a exposição dos órgãos abdominais, seguindo diretamente para a exposição do ducto biliar comum com abaixamento do duodeno, a partir da localização do fígado. 3C. Dupla ligadura do ducto biliar comum, sendo colocadas as ligaduras o mais proximal e o mais distalmente possível. 3D. Ressecção da porção do ducto biliar comum entre as duas ligaduras.

\section{Referências}

1. Poupon R, Chazouilleres O, Poupon RE. Chronic cholestatic diseases. J Hepatol 2000; 32(1):129-40.

2. Spraul AD, Cosson C, Couturier $M$, Hadchouel M, Legrand A, Lemonnier F. et al. Standard treatment of tocopherol in alagille patients with severe cholestasis is insufficient. Pediatr Res 2001; 49:232-6.

3. Sokol RRJ, Mack C. Etiopathogenesis of biliary atresia. Semin Liver Dis 2001; 21(4):517-24

4. Reichen J. Pharmacologic treatment of cholestasis. Semin Liver Dis 1993; 13:30215.

5. Cameron GR, Oakley CL. Ligation of the common bile duct. J Pathol Bacteriol 1932; 35:369-99.

6. Arresse M, Yanan MA, Suchy F. Hepatobiliary transport: molecular mechanisms of development and cholestase. Pediatr Res 2001; 44:141-7.

7. Greim H, Trülzsch D, Roboz J, Dressler K, Czygan P, Hutterer F, Schaffner F, Popper
H. Bile acids in normal rat livers and in those after bile duct ligation. Gastroenterology 1972; 63:837-45.

8. Sherlock S. Cholestasis. In: Sherlock S. Diseases of the liver and biliary system. 8ed. Boston: Blackwell Scientific Publication, 1989. p 248-72.

9. Bolder U; To-Nu HT; Schteingart CD; Frick E, Hofmann AF. Hepatocyte transport of bile acids and organics anionss in endotoxemic rats: impaired uptake and secretion. Gastroenterology 1997 112:214-25

10. Comissão de Ensino do Colégio Brasileiro de Experimentação Animal (COBEA) Manual para técnica em bioterismo; 1996.

11. Silva CRM. Efeitos da suplementação de vitamina $\mathrm{C}$ sobre efeitos decorrentes das alterações nutricionais da cirrose biliar secundária: estudo experimental em rato jovens. [Dissertação de Mestrado]. Universidade Estadual Paulista - Faculdade de Medicina de Botucatu; 2002.

12. Eduardo LHPR. Efeitos da administração de vitamina A hidrossolúvel sobre as lesões e a disfunção hepática na colestase obstrutiva: estudo experimental em ratos jovens. [Dissertação de Mestrado]. Universidade Estadual Paulista - Faculdade de Medicina de Botucatu; 2002.

13. Suzigan MI. Efeitos hepatoprotretor do extrato aquoso de picão (Bidens pilosa L.) na hepatopatia causada por colestase obstrutiva: estudo experimental em ratos jovens. [Dissertação de Mestrado]. Universidade Estadual Paulista - Faculdade de Medicina de Botucatu; 1999.

14. Santos AR. Efeitos de dietas com baixo com alto treor de gordura sobre o estado nutricional na colestase obstrutiva: estudo experimental em ratos jovens. [Dissertação de Mestrado]. Universidade Estadual Paulista - Faculdade de Medicina de Botucatu; 2003.

15. Santos AG. Estudo da correlação e regressão múltipla entre indicadores nutricionais e de lesão hepática na colestase obstrutiva crônica: estudo experimental em ratos jovens. [Dissertação de Mestrado]. Universidade Estadual Paulista - Faculdade de Medicina de Botucatu; 2003. 
Battochio APR, Santos AG, Silva, CRM, Santos AR, Coelho CAR. Adaptation of experimental model of extra-hepatic cholestasis in young rats. Acta Cir Bras [serial online] 2004 Jan-Feb;19(1). Available from URL: http://www.scielo.br/ acb.

ABSTRACT - The authors describe the adaptation of the technique of ligature and resection of the common bile duct described in the literature, emphasizing their advantages in the postnatal-surgical recovery. This work was accomplished at the Experimental Laboratory of the Department of Pediatrics of FMB-UNESP.

KEY WORDS - Cholestasis. Experimental surgery. Ligature and resection of the common bile duct.

Conflito de interesse: nenhum

Fontes de financiamento: Fapesp, Capes e CNPq

Correspondência:

Ana Paula Ronquesel Battochio

Rua José Dal Farra, 1103

18603-790 Botucatu - SP

Tel:(14)6823-5736

apbattochio@ig.com.br

Data do recebimento: $24 / 11 / 2003$

Data da revisão: $18 / 12 / 2003$

Data da aprovação: 06/01/2004

Fotos coloridas disponíveis em www.scielo.br/acb

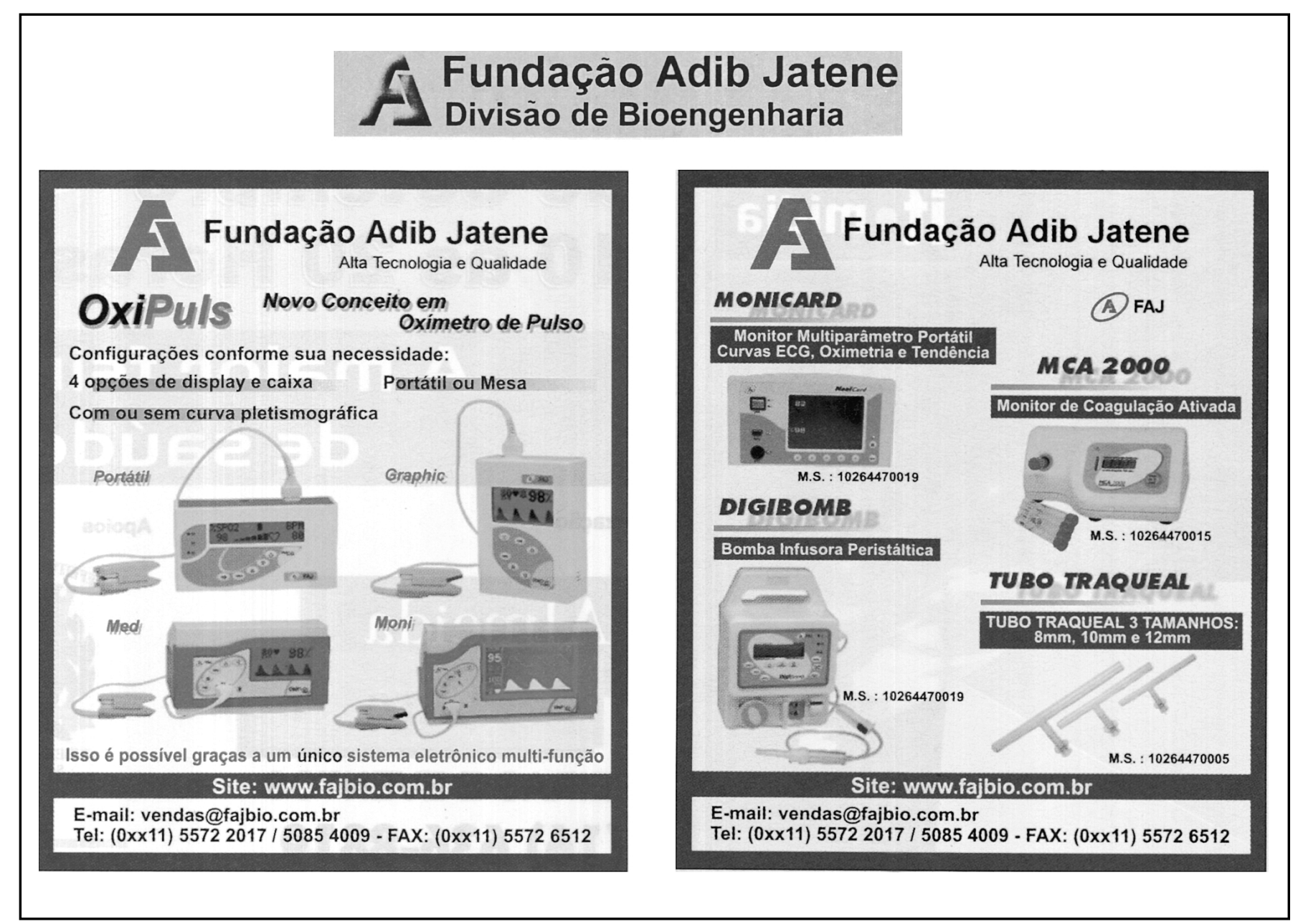

